\title{
Pulsar magnetospheres: Numerical simulations of large amplitude electron-positron oscillations
}

\author{
A. A. da $\operatorname{Costa}^{1,2, \star}$, D. A. Diver ${ }^{1}$, and G. A. Stewart ${ }^{1}$ \\ 1 Dept. of Physics and Astronomy, University of Glasgow, Glasgow G12 8QQ, Scotland, UK \\ 2 Centro de Electrodinâmica, Instituto Superior Técnico, 1049-001 Lisboa, Portugal
}

Received 21 September 2000 / Accepted 24 October 2000

\begin{abstract}
The numerical simulation of non-linear electron-positron oscillations is reported, showing the evolution of the electric field and the plasma number density for large amplitude disturbances. Sharp density gradients and changes in the oscillation frequency are demonstrated, and a new analytical framework is presented to illustrate these phenomena, particularly in the context of pulsar plasmas.
\end{abstract}

Key words. pulsars: general - plasmas

\section{Introduction}

It is a well established fact that pulsar magnetospheres must contain charged particles. Goldreich \& Julian (1969) first showed that the space surrounding an axisymmetric neutron star must contain an electron-positron (E-P) charged separated plasma, originated at the neutron star surface. Mestel (1971) and Cohen \& Toton (1971) extended this conclusion to non-axisymmetric rotators. Thus, with the same approximation, pulsar E-P magnetospheric plasmas are driven by a dipolar low-frequency strong electromagnetic wave with a high value of the magnetic induction $B$. The dominant parameter is the strength of the electromagnetic wave given by

$\eta=\frac{\mathrm{e} A_{0}}{m c}$

(Da Costa \& Kahn 1982; Da Costa 1983), where $A_{0}$ is the amplitude of the vector potential. $\eta \sim 10^{16}$ at the surface of the star, for $B \sim 10^{8} \mathrm{~T}$, and $\eta \sim 10^{6}-10^{10}$ at the speed of light cylinder (SLC) for periods $T_{\mathrm{p}} \sim 3 \mathrm{~s}-33 \mathrm{~ms}$. $\eta$ is related to the density of the electromagnetic energy $w_{\mathrm{em}} \sim 10^{23} \mathrm{Jm}^{-3}$ for $B \sim 10^{8} \mathrm{~T}$, and $w_{\mathrm{em}} \sim 10^{11} \mathrm{Jm}^{-3}$ for $B \sim 10^{2} \mathrm{~T}$, its value at the SLC for the Crab pulsar. With these values it seems reasonable to assume that the Lorentz force and its associated power $P_{\text {Lor }}$ dominates any other interaction in the process, allowing the single particle approach to be acceptable in calculating particle dynamics.

When the relativistic parameter $\gamma$ grows, the power radiated by charged particles starts to be of the same order

Send offprint requests to: D. A. Diver,

e-mail: diver@astro.gla.ac.uk

* On sabbatical leave at Glasgow. of magnitude of the Lorentz power, and the particles start to radiate high energy $\gamma$-ray photons. This has to be a single particle phenomenon, due to the $\gamma$-ray wavelength. As described in da Costa \& Kahn (1997), this is a stochastic radiative effect, to be taken into account in the equation of motion, which turns deterministic trajectories into stochastic ones, the quasi-classical regime, and the E-P plasma acquires an equivalent temperature $T_{\mathrm{eq}} \sim 10^{12} \mathrm{~K}$. As a consequence, no physical thermal effects need to be considered, and the single particle approach is reinforced.

However this strict single particle approach may be challenged if there are physical processes which develop in the rest frame, and are amplified when translated into the laboratory frame in which the neutron star is rotating. Such effects might be associated with fluctuations of the average speed and densities, due to the quasi-classical nature of the plasma flow in the laboratory frame, which creates random noise in the rest frame, duly amplified there, resulting in very strong effects back in the laboratory frame.

In fact a pulsar electric field in the laboratory frame of the order of $10^{9} \mathrm{Vm}^{-1}$ close to the SLC, turns into an electric field of the order of $10^{17} \mathrm{Vm}^{-1}$ in the rest frame (assuming a typical $\gamma \sim 10^{8}$ ). Moreover, in this rest frame, a comparatively small electric field perturbation of the order of $10^{2} \mathrm{Vm}^{-1}$ turns into a significant one in the laboratory frame, comparable with the intrinsic electric field of the rotation.

E-P plasmas are very stable to velocity perturbations in the rest frame. For example, a perturbation of $c \Delta$ transforms to a very small disturbance in the laboratory frame, in which $\gamma \gg 1$ implies $\beta \sim 1-\left(2 \gamma^{2}\right)^{-1}$, and therefore the real velocity

$v=c \frac{\beta+\Delta}{1+\beta \Delta} \approx c\left\{1-\frac{1}{2 \gamma^{2}}(1-2 \Delta)\right\}$. 
Consequently the speed of the plasma is very stable, in the sense that it is not significantly affected by collective behaviour. Plasma effects in the laboratory frame come from plasma perturbations in the rest frame, which may be taken as the co-moving plasma frame near the SLC, an acceptable approximation in the limit of large $\gamma$.

Previous simulations of E-P oscillations (Stewart 1993; Gupta 1999) suggested that density spikes evolved as an inevitable consequence of large amplitude oscillations, and that the underlying cause of such phenomena was a basic secularity present in the model. A discussion of this behaviour was presented in terms of a quasilinear analysis of the perturbed equations.

We take a different approach in this paper. The simulations here reveal a qualitative similarity in the evolution of the density, but the numerical approach used here enables any question of secularity to be avoided. The secularity observed in the earlier work (Stewart 1993; Gupta 1999) derives from the perturbative approach to the solution of the full equations, an approach which is not valid for strongly non-linear evolution. The theoretical discussion presented in this paper, describing large amplitude behaviour, together with the new numerical analysis and diagnostic insights, allows a physical understanding of the basic processes to be formulated, and applied in the context of pulsar magnetospheres.

This paper addresses the basic mathematical formulation of the E-P cold plasma oscillation in Sect. 2, in the rest frame of the initially unperturbed plasma. It then proceeds in Sect. 3 to formulate the numerical algorithm designed specifically to solve the problem. Results are also presented here, and are discussed in Sect. 4. Finally, the implications for future research are analysed in Sect. 5 .

\section{Model equations}

The conventional cold plasma equations describing plasma oscillations can be written in the form

$$
\begin{aligned}
\partial_{\mathrm{t}} n_{ \pm}+\partial_{z}\left(n_{ \pm} v_{ \pm}\right) & =0 \\
\partial_{\mathrm{t}} v_{ \pm}+\partial_{z}\left(\frac{1}{2} v_{ \pm}^{2}\right) & = \pm \frac{\mathrm{e}}{m} E \\
\partial_{z} E & =\frac{\mathrm{e}}{\epsilon_{0}}\left(n_{+}-n_{-}\right), \\
\partial_{\mathrm{t}} E & =-\frac{\mathrm{e}}{\epsilon_{0}}\left(n_{+} v_{+}-n_{-} v_{-}\right),
\end{aligned}
$$

where $\partial_{\mathrm{t}}, \partial_{z}$ denote partial derivatives in time and $z$-direction, $n_{ \pm}, v_{ \pm}$are the positron $(+)$and electron $(-)$ number density and speeds in the $z$-direction, $E$ is the electric field in the $z$-direction, $m$ is the mass of the electron, $-\mathrm{e}$ is its charge, and $\epsilon_{0}$ is the vacuum permittivity. We shall assume throughout that the oscillations will be one-dimensional, confined to $z$-direction, and aligned with any magnetic field in the plasma, so that the latter does not enter the dynamical equations. Note also that (5) and (6) are not both required, but have been quoted for completeness. The linearised form of (4) and (6) yields the familiar result:

$\partial_{\mathrm{tt}} E+\omega_{\mathrm{p}}^{2} E=0$,

where $\omega_{\mathrm{p}}^{2}=2 n_{0} \mathrm{e}^{2} /\left(\epsilon_{0} m\right)$ is the plasma frequency, $n_{0}$ being the equilibrium number density of electrons and positrons. Notice that the density equations were not used in this derivation, and that as a consequence of this fact, and the linearisation process, no spatial derivative was used. Hence, $n_{0}$ is not restricted to being spatially uniform. This means that if $n_{0}=n_{0}(z)$, then taking $E=A \exp \left(\mathrm{i} \omega_{\mathrm{p}}(z) t\right)$ leads to $n_{+}-n_{-} \propto t \partial_{z} \omega_{\mathrm{p}}$ from (5), implying that the linearised perturbation model demands that there be secular growth in the number density difference, if the plasma is spatially non-uniform (see Stewart 1992 for further details). Of course, where a uniform equilibrium is assumed, then the small-amplitude perturbation does not influence significantly the basic mass distribution, and no secular growth results.

\section{1. symmetric variables}

A better insight into the plasma dynamics can be afforded if the equations are reformulated, in order to reflect the intrinsic symmetry of this equal mass component plasma. Such a form of (3) to (6) can be constructed as follows. Taking

$$
\begin{aligned}
\Sigma & =\frac{1}{2}\left(n_{+}+n_{-}\right) L^{3}, \\
\Delta & =\frac{1}{2}\left(n_{+}-n_{-}\right) L^{3}, \\
\sigma & =\frac{1}{2}\left(v_{+}+v_{-}\right) T / L, \\
\delta & =\frac{1}{2}\left(v_{+}-v_{-}\right) T / L, \\
\epsilon & =\frac{\mathrm{e} T^{2}}{m L} E,
\end{aligned}
$$

where $L, T$ are characteristic length and time scales, then the cold plasma Eqs. (3) and (4) can be written in nondimensional form

$$
\begin{aligned}
\dot{\Sigma}+(\Sigma \sigma+\Delta \delta)^{\prime} & =0 \\
\dot{\Delta}+(\Sigma \delta+\Delta \sigma)^{\prime} & =0 \\
\dot{\sigma}+\frac{1}{2}\left(\sigma^{2}+\delta^{2}\right)^{\prime} & =0 \\
\dot{\delta}+(\sigma \delta)^{\prime} & =\epsilon,
\end{aligned}
$$

where denotes $(1 / T) \partial_{\mathrm{t}}$, and ' denotes $(1 / L) \partial_{z}$. The electric field Eqs. (5) and (6) can be cast in the form

$$
\begin{aligned}
\epsilon^{\prime} & =p \Delta, \\
\dot{\epsilon} & =-p(\Sigma \delta+\Delta \sigma),
\end{aligned}
$$

where $p=2 \mathrm{e}^{2} T^{2} /\left(\epsilon_{0} m L^{3}\right)$ is the mesh plasma frequency, squared. 
Now the small-amplitude analysis yields

$$
\begin{aligned}
\dot{\Sigma}+\left(\Sigma_{0} \sigma\right)^{\prime} & =0, \\
\dot{\Delta}+\left(\Sigma_{0} \delta\right)^{\prime} & =0, \\
\dot{\sigma} & =0, \\
\dot{\delta} & =\epsilon, \\
\dot{\epsilon} & =-p \Sigma_{0} \delta,
\end{aligned}
$$

where a subscript zero denotes the equilibrium value of a variable, and we have assumed $\Delta_{0}=\sigma_{0}=\delta_{0}=0$. This gives once more

$\delta=D \exp \left(\mathrm{i} \omega_{\mathrm{p}} t / T\right)$,

where $\omega_{\mathrm{p}}=\sqrt{p \Sigma_{0}}$, and $D$ is a constant. Notice now that if the plasma equilibrium is spatially non-uniform,

$\delta^{\prime}=\frac{\mathrm{i} \omega_{\mathrm{p}}^{\prime} t}{T} D \exp \left(\mathrm{i} \omega_{\mathrm{p}} t\right)$

showing that the derivative of the velocity difference grows with time. When (25) is substituted into (20) and (19),

$\Delta=\frac{D \Sigma_{0}}{\mathrm{i} \omega_{\mathrm{p}}}\left(\frac{\Sigma_{0}^{\prime}}{\Sigma_{0}}+\left(\mathrm{i} \omega_{\mathrm{p}} t / T-1\right) \frac{\omega_{\mathrm{p}}^{\prime}}{\omega_{\mathrm{p}}}\right) \exp \left(\mathrm{i} \omega_{\mathrm{p}} t / T\right)$,

$\Sigma=\Sigma_{0}-\sigma t /\left(\Sigma_{0}^{\prime} T\right)$,

and $\sigma$ is constant in time, with an undefined spatial variation. Hence the temporal growth in the difference between electron and positron speeds manifests itself as a secularity in the density terms, and also by either of (22) or (23) implies unconstrained growth in time of the electric field gradients, as was found earlier. (The same conclusion is reached irrespective of which of (17) or (18) is used.) However, this approach emphasises the role of the combined velocity $\sigma$ and total mass density $\Sigma$ in the evolution of the perturbation. The secular growth of the density terms $\Sigma$ and $\Delta$ is inconsistent with either particle or energy conservation and therefore the linearised treatment predicts unphysical behaviour for even modest inhomogeneity, in marked contrast to conventional electron-ion plasmas. The full non-linear evolution contains the saturation mechanism which avoids unbounded growth, in that the quadratic terms in the velocity Eqs. $(15,16)$ constrain the evolution. Then from (15), $\sigma$ cannot remain constant if $\delta$ grows in time, and therefore a limiting total velocity for the plasma must ensue, if energy conservation is to hold. This in turn ensures that there is a significant distortion of the total mass density across the perturbation, and that the electron and positron fluids are not required to be symmetrically distributed across the disturbance. However, the linear treatment does highlight that the initial growth in all quantities is fastest where the density gradient is greatest. Since the problem to be addressed in this article is the large amplitude plasma oscillation, in which the number density is significantly spatially non-uniform, we must solve the full non-linear problem numerically.

\section{Numerical approach}

\subsection{Algorithm}

The Eqs. (13-16) take the form

$\frac{\partial \mathbf{y}}{\partial t}+\frac{\partial \mathbf{f}}{\partial z}=\mathbf{g}$

where

$\mathbf{y}=\left[\begin{array}{c}\Sigma \\ \Delta \\ \sigma \\ \delta\end{array}\right]$

$\mathbf{f}=\left[\begin{array}{c}\Sigma \sigma+\Delta \delta \\ \Sigma \delta+\Delta \sigma \\ \frac{1}{2}\left(\sigma^{2}+\delta^{2}\right) \\ \sigma \delta\end{array}\right]$

$\mathbf{g}=\left[\begin{array}{c}0 \\ 0 \\ 0 \\ p \epsilon\end{array}\right]$

The electric field will be given via (17). Following Mitchell \& Griffiths (1987), we will use a Lax-Wendroff type finite difference approach to solve the above equations. Assuming discrete coordinates $z=m h, t=n k$ where $h=\Delta z, k=\Delta t$, and using the notation $\mathbf{y}(m h, n k) \equiv \mathbf{y}_{m}^{n}$, we can write

$$
\begin{aligned}
\mathbf{y}_{m}^{n+1}= & \exp \left(k \partial_{t}\right) \mathbf{y}_{m}^{n} \\
\approx & \left(1+k \partial_{\mathrm{t}}+\frac{1}{2} k^{2} \partial_{\mathrm{t}}^{2}\right) \mathbf{y}_{m}^{n} \\
= & \mathbf{y}_{m}^{n}+k\left(\mathbf{g}_{m}^{n}-\partial_{z} \mathbf{f}_{m}^{n}\right)+\frac{1}{2} k^{2} \partial_{\mathrm{t}} \mathbf{g}_{m}^{n} \\
& -\frac{1}{2} k^{2} \partial_{z}\left(\mathbf{A}\left(\mathbf{g}_{m}^{n}-\partial_{z} \mathbf{f}_{m}^{n}\right)\right)
\end{aligned}
$$

where

$\mathbf{A}=\frac{\partial \mathbf{f}}{\partial \mathbf{y}}$

is the Jacobian for the system. The electric field term $\mathbf{g}_{m}^{n}$ is evaluated directly from (17) by corrected trapezoidal integration. Also, the single component in $\partial_{\mathrm{t}} \mathbf{g}$ is replaced directly by substituting from (18). This leaves us with an explicit finite difference algorithm with which to integrate the cold plasma equations. Note that the identical approach was used to construct an algorithm based on (3-4), suitably non-dimensioned. The two approaches produced indistinguishable results. Finally, in order to smooth out noisy density fluctuations which are otherwise numerically unstable, a simple 3-point Simpson's rule was used to dampen unwanted high-frequency jitter. 


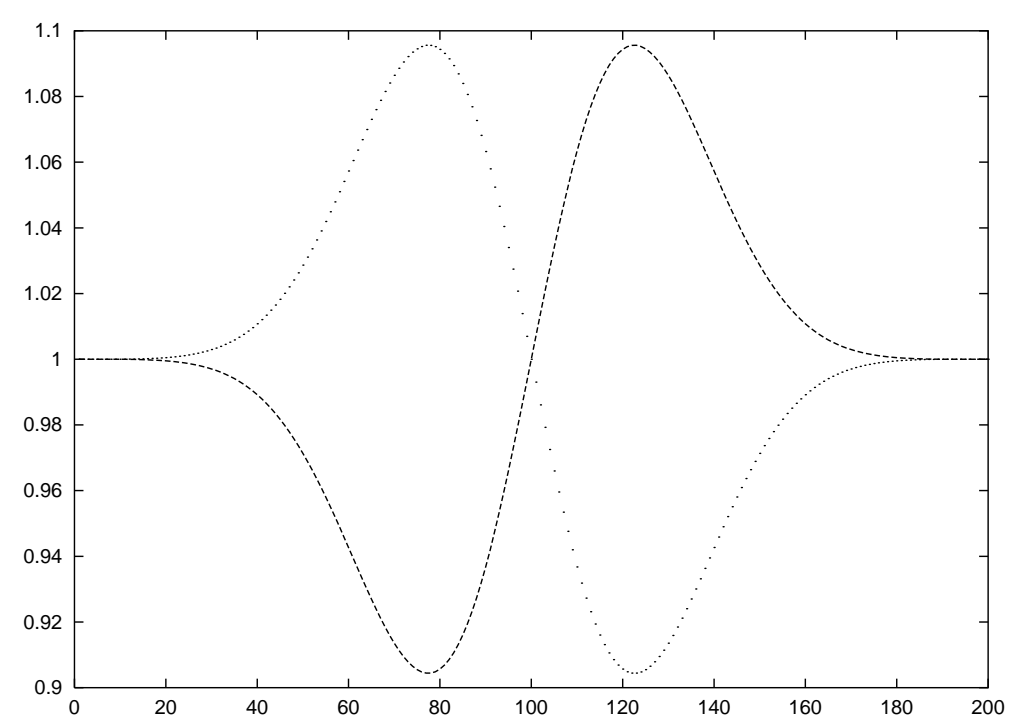

Fig. 1. Initial density profile for a $10 \%$ density perturbation $\left(n_{+}\right.$dotted, $n_{-}$dashed $)$

Table 1. Parameters for density perturbation computation

\begin{tabular}{|c|l|l|}
\hline$m m$ & 200 & number of spatial points \\
$n$ & 1500 & number of time steps \\
$h$ & 0.7 & spatial mesh increment \\
$k$ & 0.02 & temporal mesh increment \\
$\lambda$ & 2.5 & Gaussian coefficient \\
$p$ & 0.75 & mesh plasma frequency, squared \\
$m_{\mathrm{r}}$ & 90 & half-width of initial perturbation \\
\hline
\end{tabular}

\subsection{Boundary conditions}

Two approaches to boundary and initial conditions were chosen: (i) perturbing the density, and (ii) perturbing the velocity field. Perturbations are confined to a sub-range of the full spatial mesh; in other words, if the spatial range is $[1, \mathrm{~mm}]$ then the initial perturbation ranges over $\left[m m / 2-m_{\mathrm{r}}, m m / 2+m_{\mathrm{r}}\right]$. Moreover, in order to avoid sharp gradients at the edges of the initial perturbation, the form of the initial disturbance across the whole range $2 m_{\mathrm{r}}$ is multiplied by a Gaussian envelope.

\subsection{Initial density perturbation}

The initial conditions here are all speeds identically zero, and the density of electrons and positrons given by

$$
\begin{aligned}
n_{ \pm}(m h)= & 1 \pm z \exp \left(-\left(\lambda \frac{m-m^{*}}{m_{\mathrm{r}}}\right)^{2}\right) \\
& \times \sin \left(\pi \frac{m-\delta_{m}}{m_{\mathrm{r}}}\right)
\end{aligned}
$$

for $m \in\left[m^{*}-m_{\mathrm{r}}, m^{*}+m_{\mathrm{r}}\right]$, where $m^{*}=m m / 2$, and $n_{ \pm}=1$ elsewhere. The values of the numerical parameters for the calculations shown are given in Table 1.
An example of the initial density perturbation for electrons and positrons is shown in Fig. 1. All initial density profiles are identical, apart from scale. The initial electric field is calculated to be consistent with (34).

Figure 2 shows the evolution of the positron density from an initial $1 \%$ sinusoidal variation from unity, modified by the Gaussian envelope discussed above $(z=0.02)$. It is clear that the initial disturbance is not a solution of the equations, and the perturbation evolves in profile, showing a characteristic deepening where the positron density is depressed.

(Note that in all graphs, only 40 representative time steps were plotted from the actual total of $n$; this was to aid clarity in the plotting output.)

Figure 3 shows how the total plasma density $\Sigma$ behaves as a function of time, showing the tendency of the plasma mass distribution to be non-uniform in the central regions (although the maximum size of the perturbation is less than $3 \%$ ). For clarity, the actual profiles of positron and electron number densities corresponding to the last time step are displayed in Fig. 4. The accompanying electric field plot (Fig. 5) shows the regular, stable oscillation expected of small perturbations. Note that the disturbance leaks only marginally outside the range of the initial perturbation; this is the result of the diffusive term in (32) characteristic of Lax-Wendroff.

Increasing the density perturbation to $10 \%(z=0.2)$, Fig. 6 shows the onset of markedly non-linear behaviour, with the density profile showing sharp features evolving, and the electric field in Fig. 9 demonstrating a drop in the local oscillation frequency towards the centre of the disturbance, where the plasma density is substantially lower than in the initial conditions.

Note that the number density also becomes strongly localised at the edges of the oscillation; this is particularly significant in Fig. 8, where it can be seen that $\Sigma$ becomes a strong function of position. For clarity, the final electron 


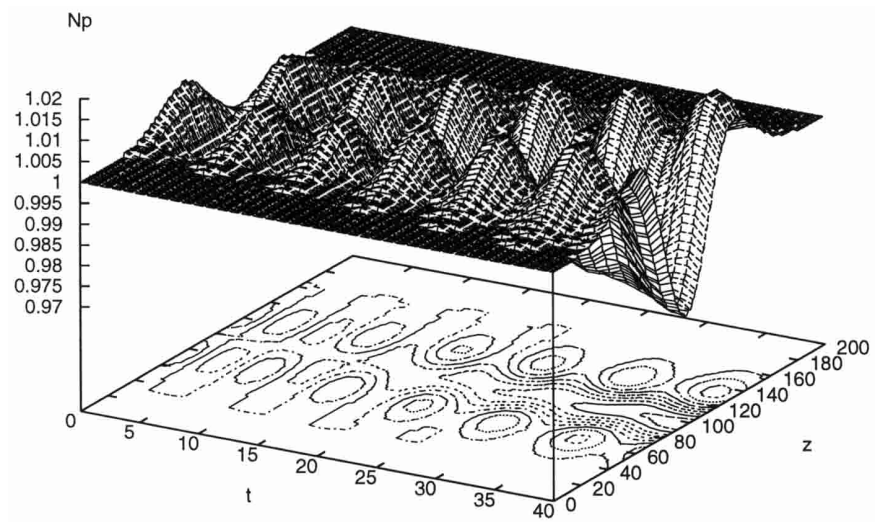

Fig. 2. Evolution of $n_{+}$after an initial $1 \%$ density perturbation

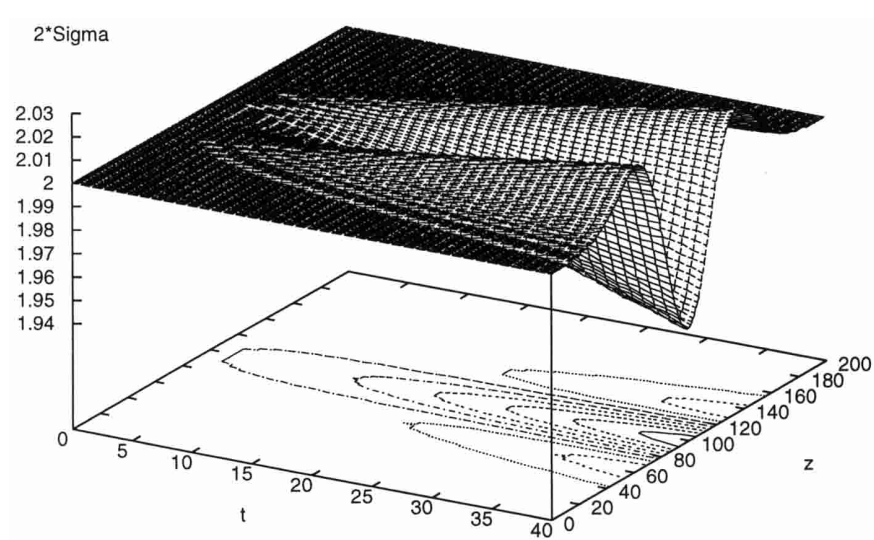

Fig. 3. Evolution of $\Sigma$ after an initial 1\% density perturbation

and positron density profiles for this case are shown in Fig. 7.

This non-linear evolution is also apparent in the velocity. Figure 10 shows the development of $\sigma$, the sum of the electron and positron velocities, as a function of time, demonstrating that there is spatial structure in $\sigma$, underlining the earlier analysis. The difference in species velocity is recorded in Fig. 11, which shows the evolution of $\delta$.

\subsection{Initial velocity perturbation}

Setting the densities to unity, and the electric field to zero, the initial velocity condition is as follows:

$$
\begin{aligned}
v_{ \pm}(m h)= & \pm z \exp \left(-\left(\lambda \frac{m-m^{*}}{m_{\mathrm{r}}}\right)^{2}\right) \\
& \times \sin \left(\frac{\pi}{2} \frac{m-\delta_{m}}{m_{\mathrm{r}}}\right),
\end{aligned}
$$

for $m \in\left[m^{*}-m_{\mathrm{r}}, m^{*}+m_{\mathrm{r}}\right]$, and $v_{ \pm}=0$ elsewhere. Note that here only half the period of the sinusoid is chosen, as shown in Fig. 12. The values of the numerical parameters for the calculations shown are given in Table 2. The evolution of the positron density for $z=0.3$ is shown in Fig. 13, in which it is evident that sharp density gradients evolve once more, and that the central area of the oscillation has
Table 2. Parameter values for velocity perturbation computation

\begin{tabular}{|c|l|l|}
\hline$m m$ & 300 & number of spatial points \\
$n$ & 1200 & number of time steps \\
$h$ & 0.7 & spatial mesh increment \\
$k$ & 0.02 & temporal mesh increment \\
$\lambda$ & 2.5 & Gaussian coefficient \\
$p$ & 0.75 & mesh plasma frequency, squared \\
$m_{\mathrm{r}}$ & 90 & half-width of initial perturbation \\
\hline
\end{tabular}

significantly low plasma density. Once more this leads to a drop in the local plasma frequency, as demonstrated in Fig. 14, where the oscillation of the electric field is clearly slower in the centre compared to the edges.

Finally, note that the physical values of fields, frequencies and speeds are determined via the mesh plasma frequency, $p$, and typical plasma parameters characterising a pulsar. For example, if we assume the required typical electron number densities for $\gamma$-ray radiation in the laboratory frame as stated in da Costa \& Kahn (1982), then in the rest frame this means taking $n_{-} \approx 10^{21} \mathrm{~m}^{-3}$, implying a plasma frequency of $\sim 10^{11} \mathrm{~s}^{-1}$. Since

$$
\begin{aligned}
p & =\frac{2 \mathrm{e}^{2}}{\epsilon_{0} m} \frac{T^{2}}{L^{3}} \\
& \approx 610^{3} \frac{T^{2}}{L^{3}} \\
& \approx 1, \quad(\text { from Tables } 1 \text { and } 2)
\end{aligned}
$$

and given that (8) implies $L \approx 10^{-7} \mathrm{~m}$ (assuming that the numerical simulations yield $\Sigma \sim O(1)$ ), then $T \sim$ $10^{-12} \mathrm{~s}^{-1}$. As a consequence, $v_{ \pm} \sim L / T \sim 10^{5} \mathrm{~ms}^{-1}$, nonrelativistic, as consistent with the model equations for the numerical frame. Note also that the maximum electric field value implied by the computations and the consequent dimensional analysis is approximately (from (12)) $10^{5} \mathrm{Vm}^{-1}$.

\section{Discussion}

The novel aspect of the simulations presented here is that the non-linear evolution of the plasma density causes a drop in the local plasma frequency, which in turn affects the onward evolution of the electric field oscillation as a function of position across the disturbed region. This frequency drop in the centre of the oscillation region causes a phase mismatch with the periodic motion of the chargedparticle densities, causing further evacuation of the plasma from the slowly changing electric field regions, relocating it to the more rapidly changing edges. In this way, sharp density features evolve, and eventually the mesh resolution is defeated.

The essential nonlinearity in the plasma evolution comes from magnitude of the phase lag between the edges 


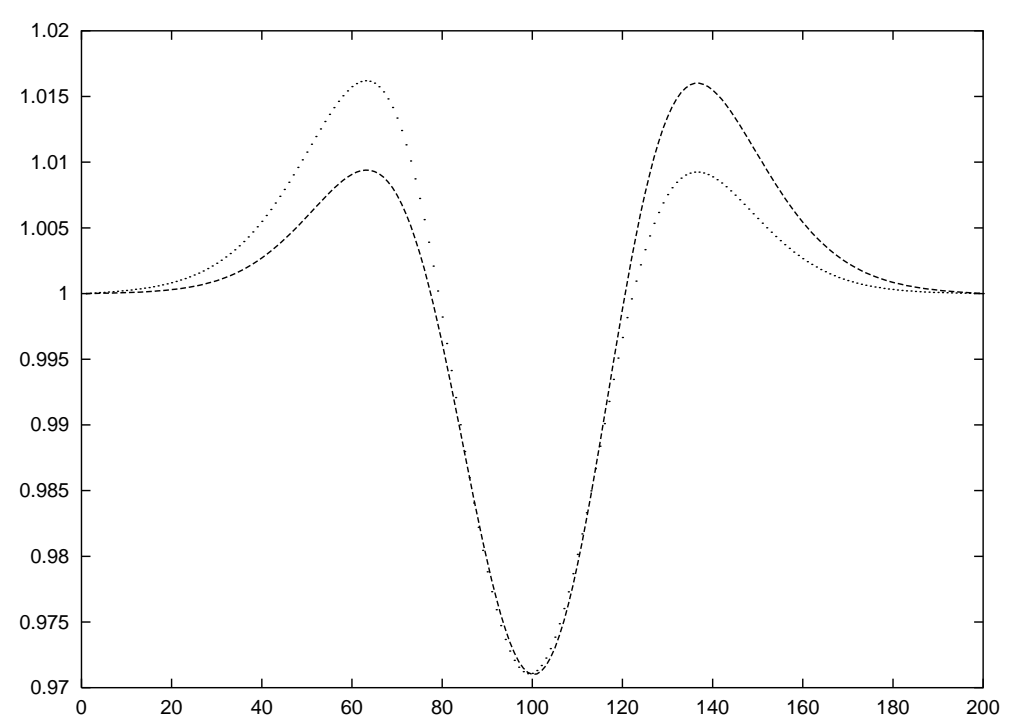

Fig. 4. Profiles of $n_{+}$(dotted) and $n_{-}$(dashed) for the last time step, after an initial $1 \%$ density perturbation

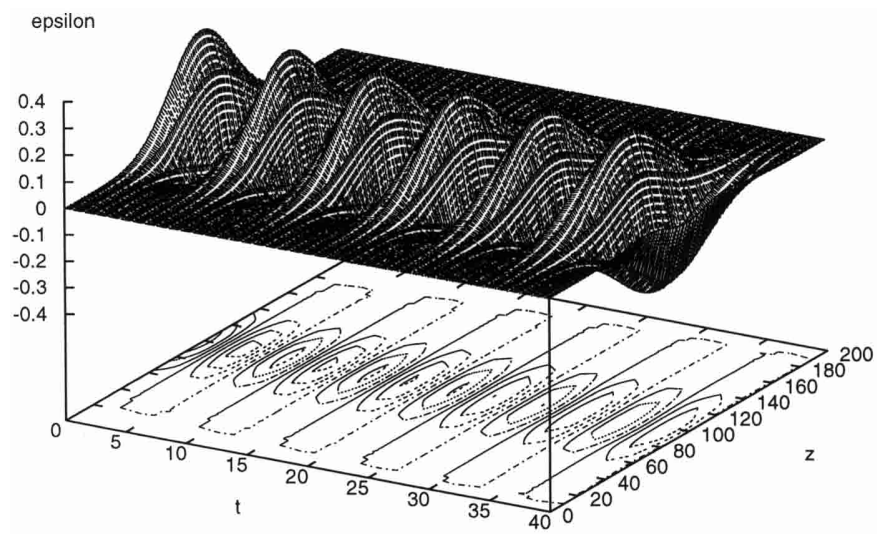

Fig. 5. Evolution of $\epsilon$ after an initial 1\% density perturbation

$$
\mathrm{Np}
$$

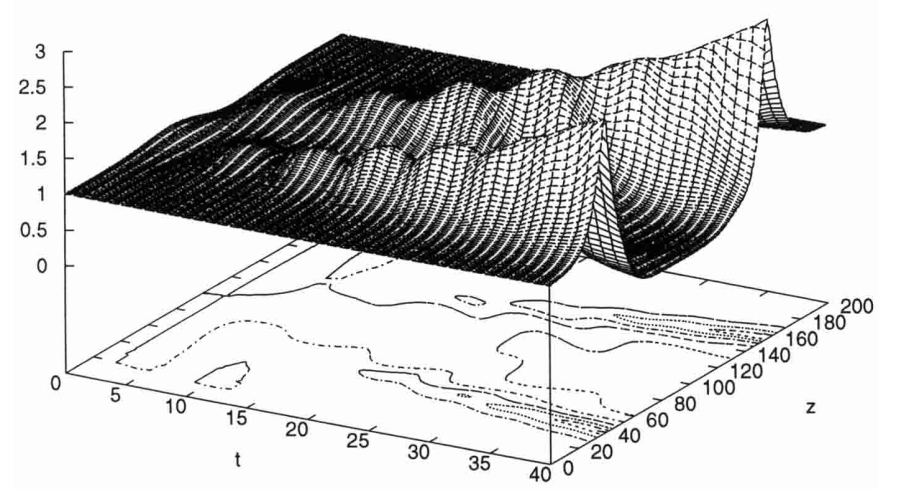

Fig. 6. Evolution of $n_{+}$after an initial $10 \%$ density perturbation

and centre of the oscillation. A large phase difference requires a significant change in plasma frequency at the centre of the disturbance compared to wings, and consequently, the plasma density must be substantially restructured in order to achieve this. The kinetic energy necessary to transport the plasma away from the central areas is derived either from the initial electrostatic energy input or from the initial velocity distribution, prescribed in the starting conditions for the computation. This explains why the extent of the nonlinearity present in the evolution scales with the initial amplitude of the disturbance.

Note that the cold plasma equations have no thermodynamics which can limit the particle density, and so to this extent the unlimited growth in density is an artifice of the model equations. This also explains why the oscillation remains localised, and does not leak out of the initially perturbed region (other than by numerical diffusion): it is simply a lack of both a sound speed and a Debye length in the cold plasma model. A more advanced model would incorporate the requisite thermal effects which would allow the densities to saturate in a physically acceptable way; such a modelling approach is currently being considered by the authors.

\section{Considerations for the future}

The calculations presented here are confined to the plasma rest frame, and to 1-D electrostatic oscillations. We must consider how to extend these calculations to a physically more acceptable model, still in the context of the rest frame, followed by generalisation of their implications to the laboratory frame.

The current simulations show that density enhancement does happen within this context, suggesting that electron-positron interactions could become significant physical processes at the edges of such oscillations. Given the nature of the pulsar plasma environment, the high energy photons produced by such processes may well escape the pulsar and be detected directly in the laboratory frame, or may possibly exchange momentum with the plasma a whole, and produce further particle pairs.

Moreover, the isolated nature of these oscillations offers the possibility of stochastically accelerating charged particles, drawn from the high-energy tail of the plasma 


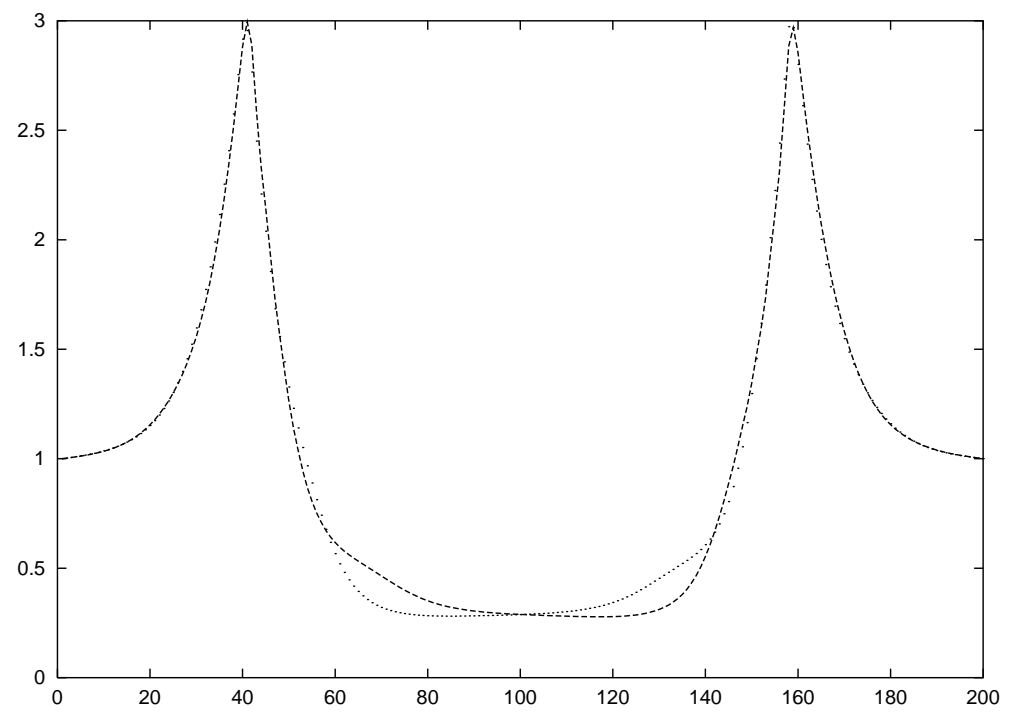

Fig. 7. Profile of $n_{+}$(dotted) and $n_{-}$(dashed) for last time step, after an initial $10 \%$ density perturbation

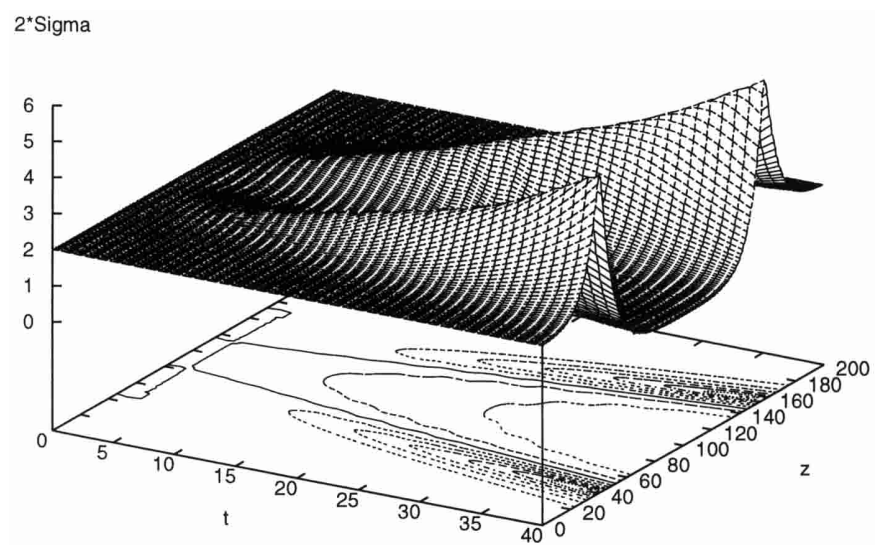

Fig. 8. Evolution of $\Sigma$ after an initial $10 \%$ density perturbation

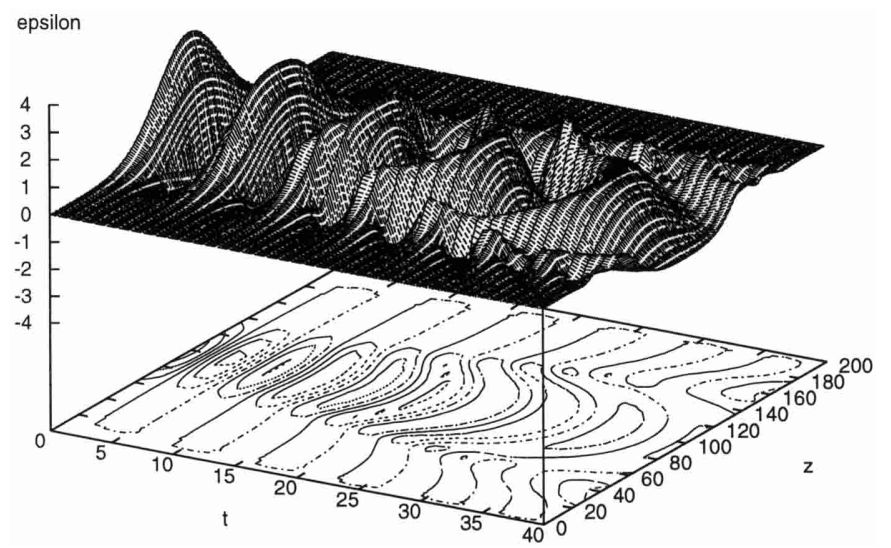

Fig. 9. Evolution of $\epsilon$ after an initial $10 \%$ density perturbation

particle distribution function, through particle interactions with randomly phased moving electric fields scattered throughout the neutron star plasma. Such a Fermi process could accelerate a population of particles to energies otherwise inaccessible by standard modelling in this context, and may afford a possible escape from the

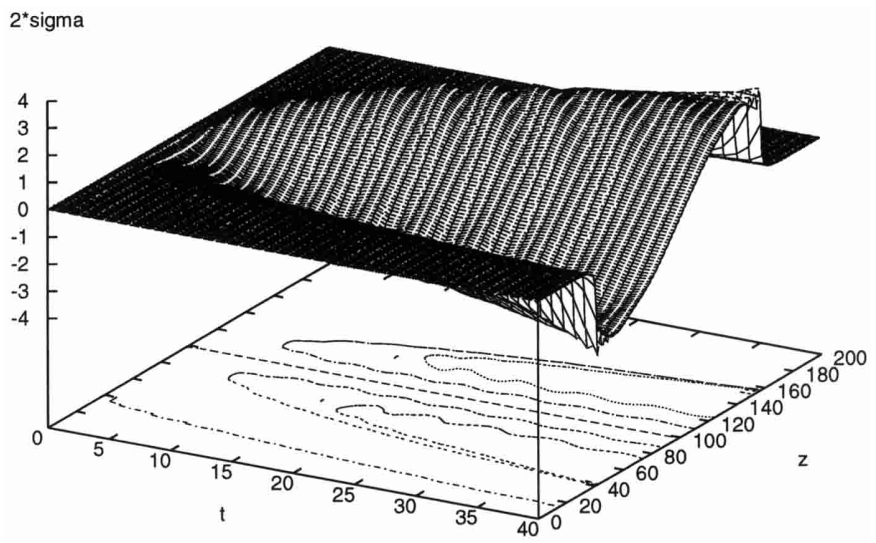

Fig. 10. Evolution of $\sigma$ after an initial $10 \%$ density perturbation

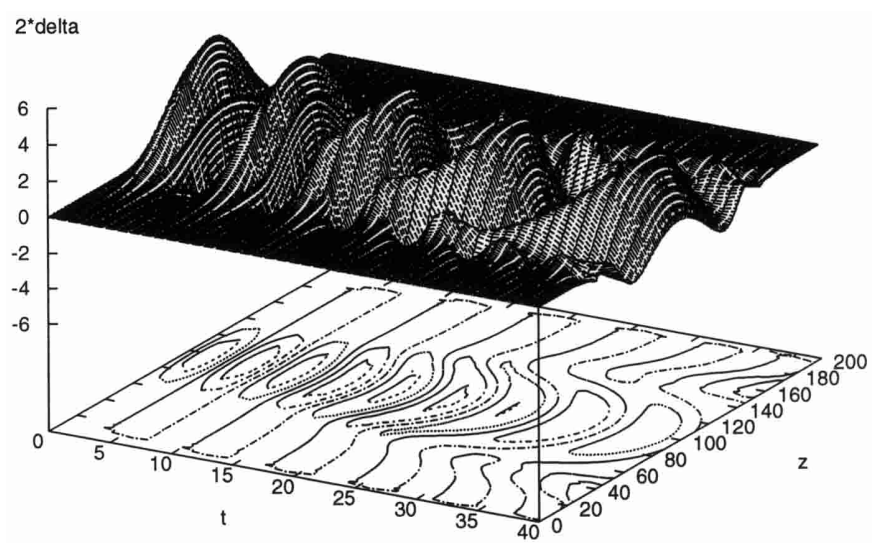

Fig. 11. Evolution of $\delta$ after an initial $10 \%$ density perturbation

high-energy cut-off of radiation suggested by the single particle approach. Note that the calculated peak electric field on the non-linear plasma oscillations $\left(\sim 10^{5} \mathrm{Vm}^{-1}\right)$, when translated back to the laboratory frame, is much 


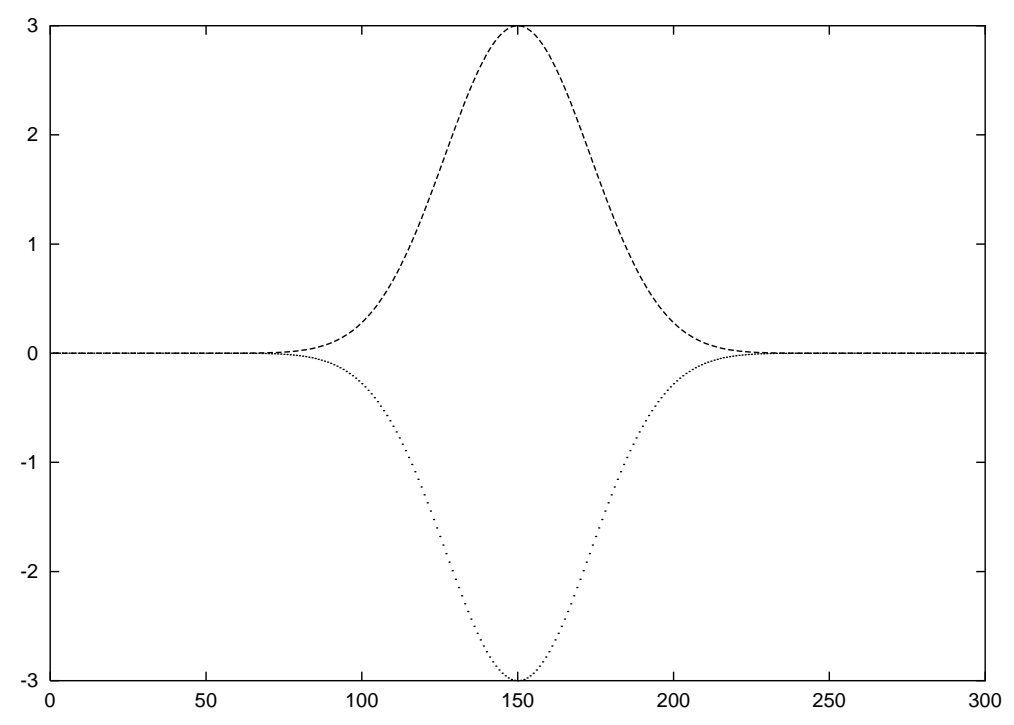

Fig. 12. Initial profiles of $v_{+}$(dashed) and $v_{-}$(dotted), for $z=0.3$

$$
\mathrm{Np}
$$

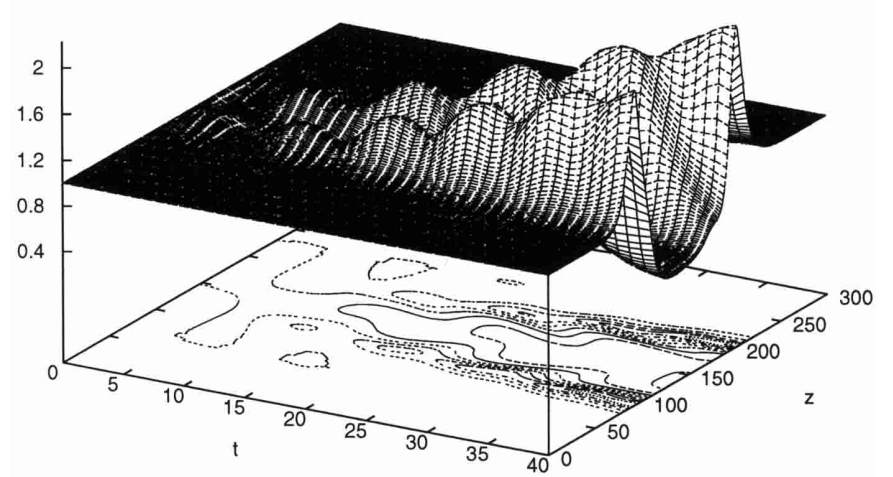

Fig. 13. Evolution of $n_{+}$after an initial $z=0.3$ velocity perturbation

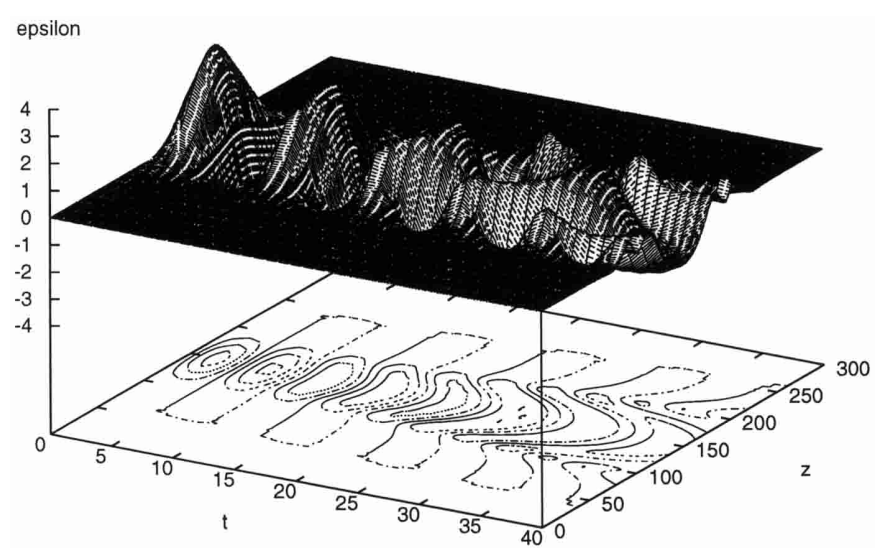

Fig. 14. Evolution of $\epsilon$ after an initial $z=0.3$ velocity perturbation

greater that the typical low-frequency dipolar field, albeit restricted to localised plasma oscillations. This reinforces our contention that collective effects do have an important influence on the overall electrodynamics of the pulsar.

The present work neither takes into account the presence in the rest frame of the transformed pulsar low frequency electromagnetic wave, nor more general electromagnetic effects, when

$\partial_{\mathrm{t}} \mathbf{E}+\frac{\mathrm{e}}{\epsilon_{0}}\left(n_{+} \mathbf{v}_{+}-n_{-} \mathbf{v}_{-}\right) \neq \mathbf{0}$.

These rest frame properties have also to be transformed into the laboratory frame, with the inherent consequences assessed.

Particle pseudo-trajectories in the context of the single particle approach, calculated close to the speed of light cylinder (SLC) using the mathematical rotating frame of the star, show that due to radiative effects they have a Fourier spectrum with a fundamental frequency in the $\mathrm{MHz}$ domain (da Costa \& Kahn 1997). This is not a problem since the dipolar EM field of the pulsar used in the calculations is centered in the $200 \mathrm{~Hz}$ frequency, and the two spectra cannot overlap.

However the EM field of the star is not purely dipolar, containing not only a component generated by rotating charge and current density distributions created by the plasma motion itself (da Costa \& Kahn 1982), but also a component arising directly from the rest frame effects. This extended EM field has a spectrum that is an extension of the spectrum discussed by da Costa \& Kahn (1985) and which overlaps with the trajectory spectrum, and therefore resonant effects must exist between the two.

On the other hand, the plasma radiates curvature radiation. When stochastic radiative effects are taken into account, with the radiation of high-energy gamma-ray photons, such radiation has to arise from individual particles, which ensures its incoherence. However the power radiated by individual particles might be affected by collective phenomena. In low frequency radiation (Boyd \& Sanderson 1969) the broadening of emission lines due to the action of the plasma as a whole, means we have to substitute

$P(\omega, \mathbf{p}, \mathbf{r}, t)=\int_{-\infty}^{+\infty} P\left(\omega_{1}, \mathbf{p}, \mathbf{r}, t\right) \delta\left(\omega-\omega_{1}\right) \mathrm{d} \omega_{1}$ 
the radiated power spectrum by individual particles by

$P^{\mathrm{I}}(\omega, \mathbf{p}, \mathbf{r}, t)=\int_{-\infty}^{+\infty} P\left(\omega_{1}, \mathbf{p}, \mathbf{r}, t\right) \Lambda\left(\omega-\omega_{1}\right) \mathrm{d} \omega_{1}$

where $\Lambda(\omega)$ is the line profile function associated mostly with collisional broadening and Doppler broadening. In the case of relativistic plasmas $\Lambda(\omega)$ is the result of physical processes to be identified.

For example in the case of radiative relativistic plasmas the distribution function has a $T_{\text {eq }} \sim 10^{12} \mathrm{~K}$ as seen, but it is not centered in $\mathbf{p}=\mathbf{0}$. All calculations so far done (da Costa \& Kahn 1997) use the radiation of individual particles without collective corrections, and therefore $P(\omega, \mathbf{p}, \mathbf{r}, t)$. However, there must exist a Doppler broadening effect which transforms $P(\omega, \mathbf{p}, \mathbf{r}, t) \rightarrow P^{\mathrm{I}}(\omega, \mathbf{p}, \mathbf{r}, t)$, due to the presence of $T_{\text {eq }}$. But the distribution function and therefore $T_{\text {eq }}$ depends on the characteristics of the power spectrum law, and now such a power spectrum depends on the distribution function, a very difficult stochastic functional problem.

This problem is even more complicated when it is realised that:

- The distribution function must depend on the nonlinear processes which happen in the rest frame translated into the laboratory frame;

- These non-linear processes are triggered by noise associated with the radiation of the high-energy gammaray photons, creating an imbalance in plasma density and speed, and depend on the rate of radiation of photons characteristic of $P^{\mathrm{I}}$;

- The characteristics of $P^{\mathrm{I}}$ may change with the Fermi acceleration, and the distribution function with electron-positron annihilation and production.

This original paper has begun to address the fundamental role of collective effects in the overall physical description of pulsar phenomena. Although the physical processes in the rest frame may be low energy, they turn out to be very significant in the high energy laboratory frame environment, giving a new meaning to the single particle approach. We propose then that the single particle approach survives, but in a modified form, through the effects of stochastic collective phenomena. For example, the strict single particle radiative power spectrum will be altered by the influence of collective acceleration mechanisms. Such classical and quasi-classical related problems require new approaches to establish the characteristics of the plasma flow in pulsar magnetospheres, to be able to explain the associated radiative mechanisms.

Acknowledgements. The authors thank Professor E. W. Laing for the stimulating discussions on the theory of electronpositron plasmas and its application to pulsar modelling.

AAdaC sabbatical leave was sponsored by Fundacão para a Ciência e Tecnologia, Portugal. We thank Instituto Superior Técnico for granting the sabbatical leave.

The authors are grateful for facilities provided via PPARC grant GR/L56510.

\section{References}

Boyd, T. J. M., \& Sanderson, J. J. 1969, Plasma Dynamics (Thomas Nelson \& Sons Ltd, London)

Cohen, J. M., \& Toton, E. T. 1971, Pulsar Electrodynamics, Astrophys. Lett., 7, 213

da Costa, A. A. 1983, MNRAS, 204, 1125

da Costa, A. A., \& Kahn, F. D. 1982, MNRAS, 199, 211

da Costa, A. A., \& Kahn, F. D. 1985, MNRAS, 215, 701

da Costa, A. A., \& Kahn, F. D. 1991, MNRAS, 251, 681

da Costa, A. A., \& Kahn, F. D. 1997, MNRAS, 284, 1

Goldreich, P., \& Julian, W. H. 1969, ApJ, 157, 869

Mestel, L. 1971, Nat. Phys. Sci., 233, 149

Mitchell, A. R., \& Griffiths, D. F. 1987, The Finite Difference Method in Partial Differential Equations (John Wiley \& Sons, Chichester, UK)

Gupta, S. S., \& Kaw, P. K. 1999, Phys. Rev. Lett., 82, 1867

Stewart, G. A. 1993, J. Plas. Phys., 50, 521 